\title{
ANATOMÍA Y ANÁLISIS CUALITATIVO DE SAPONINAS, RESINAS Y ACEITES EN LOS ÓRganos de PASCALIA GLAUCA (AsteraceAe)
}

\author{
ANATOMY AND QUALITATIVE ANALYSIS OF SAPONINS, RESINS, AND OILS IN \\ organs of PAscalia gLAUCA (AsteraceAe)
}

Laboratorio de Morfología Comparada de Espermatófitas (LAMCE), Facultad de Ciencias Agrarias y Forestales, Universidad Nacional de La Plata, 60 y 119, C.C. 31 (1900) La Plata. Argentina

*anaramba@yahoo.com.ar

Citar este artículo HERNÁNDEZ, M. P. \& A. M. ARAMBARRI. 2019. Anatomía y análisis cualitativo de saponinas, resinas y aceites en los órganos de Pascalia glauca (Asteraceae). Bol. Soc. Argent. Bot. 54: 313-323.

DOI: http://dx.doi. org/10.31055/1851.2372.v54. n3.25356

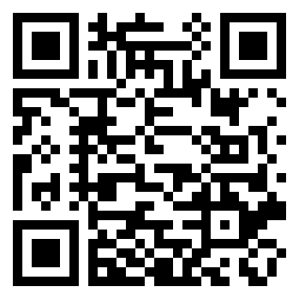

Recibido: 23 Marzo 2019 Aceptado: 14 Mayo 2019 Publicado: 30 Septiembre 2019 Editora: Ana María Gonzalez (D)

\author{
Marcelo P. Hernández y Ana M. Arambarri* (iD
}

\section{SUMMARY}

Background and aims: Pascalia glauca is a toxic weed for cattle, with wide distribution in Argentina. The toxicity is produced by an atractiloside, but the species also contains other compounds such as saponins, resins and oils with toxic properties. The aims of this work were: 1 - to perform a qualitative analysis of saponins in the underground (rhizomes + roots) and aerial organs during the four seasons of the year; 2- to study the anatomy of the vegetative and reproductive organs in search of secretory structures; 3- to identify resins and oils through histochemical tests.

M\&M: In each season of the years 2016-2017 full plants were collected. This material was used to study anatomy by conventional techniques; determine saponins and identify resins and oils by histochemical tests.

Results: Saponins were found in all organs in small quantities and their content showed variability with the season of the year, a result that is expressed in terms of average values of temperature and rainfall. The secretory ducts with resins and oils were located in the rhizomes, stems, leaves and inflorescences, not in root.

Conclusions: According to the results, all these compounds act as toxic during the year, principally in spring and increase in the summer-autumn period, due to the abundance of essential oils in the inflorescences, a characteristic that would indicate it as a promising species for its use in the control of agricultural pests.

\section{KEY WORDS}

Asteraceae, inflorescence, leaf, Pascalia glauca, reservories, root-rhizome, stem.

\section{RESUMEN}

Introducción y objetivos: Pascalia glauca es una maleza tóxica para el ganado, con amplia distribución en la Argentina. La toxicidad es producida por un atractilósido, pero la especie también contiene compuestos como las saponinas, resinas y aceites con propiedades tóxicas. Los objetivos de este trabajo fueron: 1- realizar un análisis cualitativo de saponinas en los órganos subterráneos (rizomas + raíces) y aéreos durante las cuatro estaciones del año; 2- estudiar la anatomía de los órganos vegetativos y reproductivos en busca de estructuras secretoras; 3- identificar resinas y aceites mediante pruebas histoquímicas.

M\&M: En cada estación de los años 2016-2017 se recolectaron plantas completas. Este material se usó para estudiar la anatomía mediante técnicas convencionales; determinar saponinas e identificar resinas y aceites mediante pruebas histoquímicas.

Resultados: Las saponinas se encontraron en todos los órganos en escasa cantidad y su contenido mostró variabilidad con la estación del año, resultado que se expresa en función de los valores medios de temperatura y lluvias. Los conductos secretores con resinas y aceites se localizaron en los rizomas, tallos, hojas e inflorescencias, excepto en la raíz.

Conclusiones: De acuerdo con los resultados, todos estos compuestos actúan como tóxicos durante todo el año pero principalmente en primavera y se incrementa en el período de verano-otoño por la abundancia de aceites esenciales en las inflorescencias, característica que además la indicaría como una especie promisoria para su utilización en el control de plagas agrícolas.

Palabras clave

Asteraceae, hoja, inflorescencia, Pascalia glauca, raíz-rizoma, reservorios, tallo. 


\section{INTRODUCCIÓN}

Pascalia glauca Ortega [= Wedeliaglauca (Ortega) O. Hoffm. ex Hicken] (Heliantheae, Asteraceae), es conocida como "sunchillo", "suncho", "yuyo sapo", "asolador", "carne de perro", "catay", "chilquilla", "chilquina", "chilca amarilla", "chilca amarga", "clavel amarillo", "clavel asolador", "clavelillo", "espanta colono", "espanta perro", "flor amarilla", "flor de sapo", "mata sapo", "pianta colono", "seca tierra" o "yuyo sanjuanino" (Burkart \& Carera, 1953; Marzocca, 1957; De la Peña \& Pensiero, 2004; Troiani, 2016). Esta especie americana es de amplia distribución en la Argentina, creciendo desde el nivel del mar hasta 2000 msnm y desde Jujuy hasta Chubut (Cabrera, 1963; Marzocca et al., 1986; Zuloaga et al., 2008). Es una hierba perenne, de $0,30-1,00 \mathrm{~m}$ de altura, con ciclo estival, comienza a vegetar a fines de invierno-primavera, florece en verano y fructifica en otoño, constituye una maleza invasora de difícil extirpación y tóxica (Marzocca, 1957; Cabrera \& Burkart, 1974; Boelcke, 1981; Ragonese \& Milano, 1984; Marzocca et al., 1986). Es agresiva y persistente por la presencia de rizomas, cuya formación fue develada por Burkart \& Carera (1953); posteriormente, Montaldi et al. (1984), en condiciones de laboratorio, establecieron que la alta concentración de sacarosa o de nitrato de amonio en el substrato de cultivo, estimula la formación de rizomas en la plántula. En la Argentina el primero que hizo referencia a la toxicidad fue Hieronymus (1882) y fue declarada plaga de la agricultura por decreto de fecha 22 de agosto de 1932 y su lucha está sujeta a las disposiciones de la ley 4863 de defensa agrícola (Marzocca, 1957; Marzocca et al., 1986; Troiani, 2016), resultando una de las plantas tóxicas más importantes del país, causando la muerte en bovinos, equinos, caprinos y porcinos (Gallo, 1979; Ragonese \& Milano, 1984; Correa et al., 2017). La toxicidad fue atribuida a una resina tóxica por Burkart \& Carera (1953). Platanow \& López (1978), encontraron un principio hidrosoluble hepatotóxico y consideraron que la toxicidad podría ser consecuencia de la acción conjunta de una resina y aceite, un principio hidrosoluble venenoso hepatotóxico y la presencia de saponinas. Schteingart \& Pomilio (1984), encontraron que el principio tóxico de Pascalia glauca era un diterpeno denominado atractilósido que es una sustancia tóxica con estructura similar al carboxiatractilósido que actúa inhibiendo la fosforilación oxidativa a nivel de las mitocondrias en los hepatocitos y produce una insuficiencia hepática aguda. Marzocca et al. (1986), señalaron que posiblemente el tóxico este localizado en la resina y el aceite esencial que posee. Gallo (1987), consideró de importancia para su toxicidad, la presencia de saponinas. Casabuono \& Pomilio (2000), identificaron químicamente en la misma especie, un diterpeno tetracíclico monoglicosidado, acilado y sulfatado causante de paro respiratorio en ratones. Este compuesto sería coincidente con el diterpenoide denominado atractilósido identificado por Schteingart \& Pomilio (1984). Micheloud \& Odriozola (2012), en su actualización sobre intoxicación provocada por Wedelia glauca, atribuyeron la acción tóxica al carboxiatractilosido. Mientras que Reid Lorna \& Lagomarsino (2009) y Giménez (2017), atribuyeron la toxicidad a las resinas existentes en las partes vegetativas.

Pascalia glauca se caracteriza por su fuerte aroma producido por los aceites esenciales. Hasta el presente existen pocos estudios sobre los aceites esenciales de esta especie, entre ellos Bailac et al. (2005) establecieron que es rico en limoneno, sabineno y $\alpha$-pineno. Otros autores (Rufinnengo et $a l ., 2005)$, ensayaron el efecto repelente y acaricida de los aceites esenciales de $P$. glauca. Si bien $P$. glauca se caracteriza por su toxicidad, Del Vitto \& Petenatti (2015), señalaron que también podría tener propiedades como planta medicinal y aromática.

Las características de $P$. glauca, mencionadas en los párrafos anteriores, justifican el presente trabajo cuyos propósitos son: 1- establecer la presencia y estimar de acuerdo a la escala de Harborne (1989) la abundancia de saponinas en raíz-rizoma, tallo, hojas e inflorescencias; 2- establecer la variación del contenido de saponinas en cada órgano en las diferentes estaciones del año; 3- analizar la anatomía de las partes vegetativas y reproductivas (inflorescencia) de la planta en busca de estructuras secretoras y 4- localizar las resinas y aceites en los órganos haciendo uso de reacciones colorimétricas histoquímicas.

\section{Materiales y Métodos}

Plantas frescas y completas (Fig. 1), se recolectaron en los años 2016 y 2017 en los humedales de La Balandra, Berisso, Buenos Aires, Argentina 


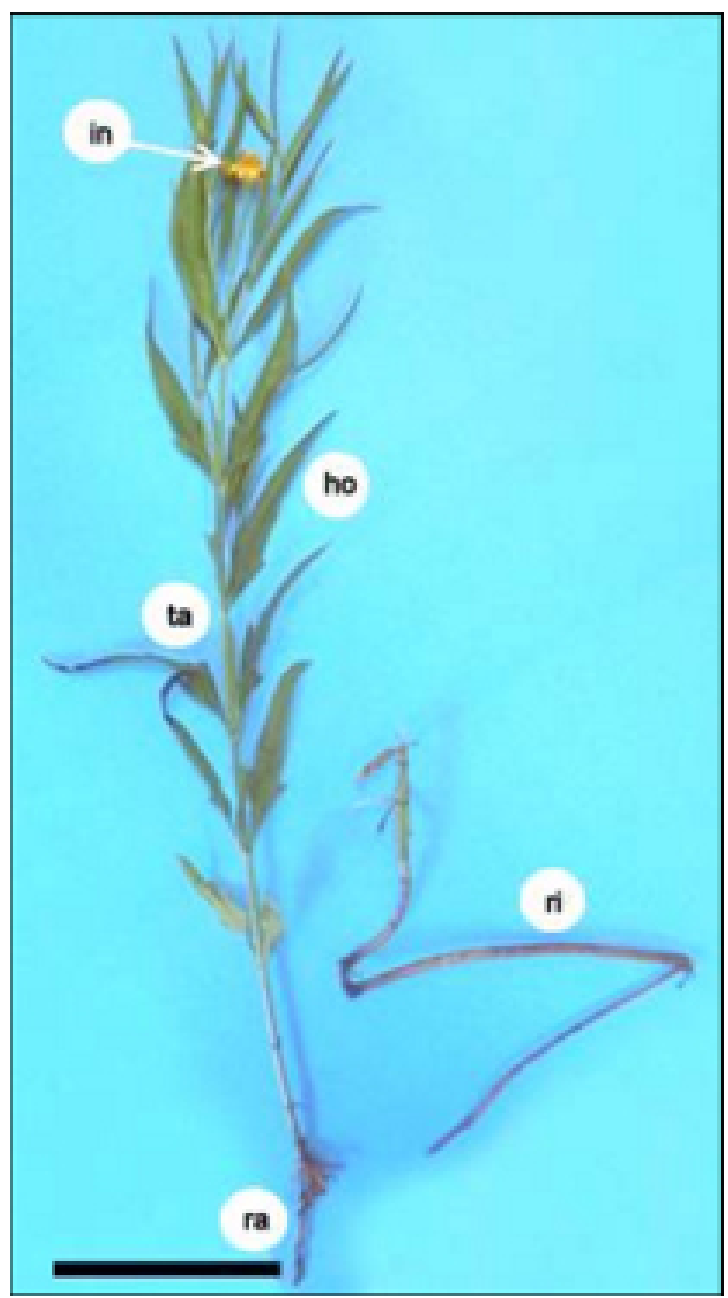

Fig. 1. Partes de la planta de Pascalia glauca. Abreviaturas = ho: hoja; in: inflorescencia; ra: raíz; ri: rizoma; ta: tallo. Escala $=10 \mathrm{~cm}$.

(34 53’ 49.40" lat. S, 57 48' 22.82" long. O). Parte del material se fijó, para su conservación y posterior estudio, en solución de formol-ácido acético glacial-alcohol etílico (FAA 70\%) (Johansen, 1940). Ejemplares de referencia se depositaron en el herbario de la Facultad de Agronomía de La Plata (Hernández 222, 223, LPAG). Para el estudio de saponinas en las cuatro estaciones del año, el material se recolectó al atardecer, se lavó para retirar las partículas de suelo y se fraccionó. Dentro de las 24 horas se determinaron las saponinas. Para ello, se pesaron $10 \mathrm{~g}$ de los órganos, raíz-rizoma, tallo, hoja, inflorescencia (Fig. 2A-D). En cada caso se molió la muestra en $100 \mathrm{~mL}$ de agua destilada (AD). El producto triturado se hirvió a baño María durante $10 \mathrm{~min}$. Una vez frio se filtró y el líquido de filtrado se enrasó a $100 \mathrm{~mL}$ con AD. Para cada uno de los órganos, de los $100 \mathrm{~mL}$ preparados, se tomaron alícuotas crecientes de 1 a $10 \mathrm{~mL}$ y vertieron en diez tubos de ensayo (Tabla 1). Una vez preparadas las diluciones, los tubos se agitaron en sentido vertical durante $15 \mathrm{~s}$, después de $15 \mathrm{~min}$ se observó la persistencia de la columna de espuma. Para evaluar cualitativamente el contenido de saponinas se utilizó la escala de Harborne (1989), que establece de acuerdo a la altura de la columna de espuma: - menor a $1 \mathrm{~cm}$, prueba ligeramente positiva. - igual a $2 \mathrm{~cm}$, prueba positiva.

- mayor a $2 \mathrm{~cm}$, prueba fuertemente positiva.

Los datos meteorológicos fueron proporcionados por el personal del Departamento de Sismología e Información Meteorológica, Facultad de Ciencias Astronómicas y Geofísicas, Universidad Nacional de La Plata (Tabla 2). Para el estudio de la epidermis se diafanizaron las hojas (Arambarri, 2018) y para la búsqueda de las estructuras secretoras se realizaron cortes transversales a mano alzada de los órganos vegetativos. Los cortes fueron decolorados con hipoclorito de sodio (50\%), lavados con agua destilada, coloreados con safranina alcohólica (80\%) o se realizó doble coloración Azul Alcian y safranina. Tanto las diafanizaciones como los cortes fueron montados en gelatina-glicerina y sellados con esmalte de uñas. Algunas de las secciones transversales así como filarios, pálea, flores fueron clarificados con hipoclorito de sodio (50\%), se usaron para las pruebas histoquímicas, empleando para la identificación de sustancias lipofílicas: Oil Red "O" (Gurr, 1971), la presencia de lípidos quedó indicada por el color rojo (paredes impregnadas con suberina, cutina), en el caso de los aceites se observaron las gotas de aceite coloreadas de rojo. Para la identificación de resinas se usó solución de cobre (Cosa et al., 2014), cuyo test es positivo cuando la resina toma coloración verde esmeralda con ligera tonalidad amarillenta. Las fotografías macroscópicas fueron tomadas con lupa Wild M8 y cámara Sony DSC-W30. El microanálisis y microfotografías fueron realizados con un microscopio óptico Gemalux XSZ-H equipado con cámara Motic y software Motic Image Plus $2.0 \mathrm{y}$ con Nikon E200 LED, con cámara digital y software Micrometrics SE Premium. 


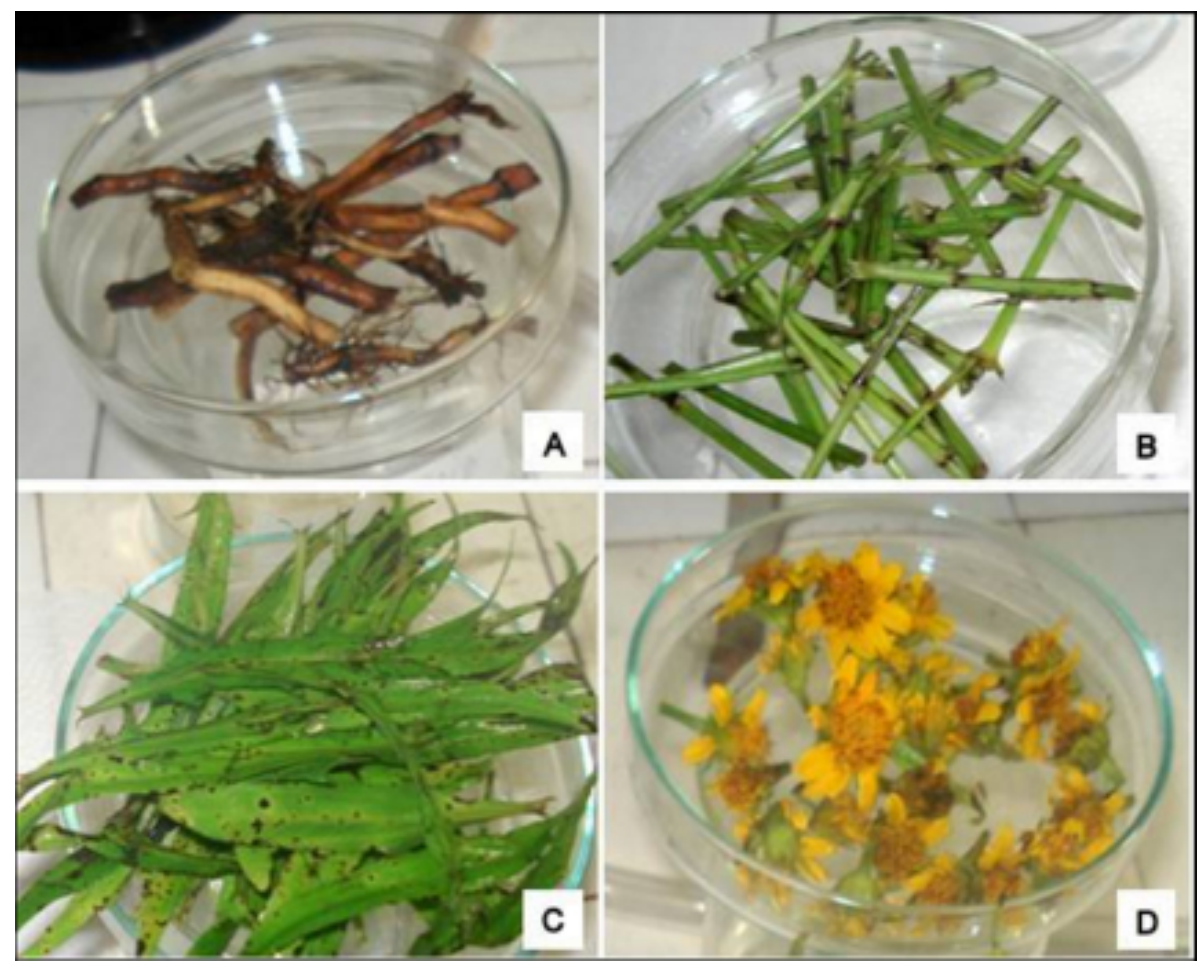

Fig. 2. Órganos fraccionados de Pascalia glauca. Diez gramos en cajas de Petri de $10 \mathrm{~cm}$ diámetro para determinar saponinas. A: Rizomas con raíces. B: Tallos. C: Hojas. D: Inflorescencias.

\section{Resultados}

\section{Compuestos químicos analizados}

Saponinas: Durante todo el año, en todos los órganos estudiados, el valor de altura de la columna de espuma fue inferior a $1 \mathrm{~cm}$; el máximo se produjo en las hojas, con valores que oscilaron entre 5 y $7 \mathrm{~mm}$ en verano-otoño y en primavera, respectivamente. En los tallos el máximo fue de $4 \mathrm{~mm}$ en verano-otoño y en rizomas $4 \mathrm{~mm}$ en otoño-invierno-primavera, mientras en las inflorescencias alcanzó 3-4 mm en verano (Tabla 2; Fig. 3A).

Resinas y aceites: en los rizomas, tallos y hojas, no así en la raíz, se hallaron reservorios conteniendo resinas y gotas de aceite; en las inflorescencias se observaron estos reservorios con abundantes aceites esenciales (Fig. 3B-D).

\section{Estructura de los órganos vegetativos}

Raíz: analizada con un diámetro 0,3-0,5 $\mathrm{mm}$, presenta rizodermis de células irregulares y exodermis con células hexagonales y paredes suberificadas rodeando a 6-8 capas de parénquima cortical, a veces con algunas cámaras aerenquimáticas; la capa más interna, la endodermis con banda de Caspary, circunda el periciclo, por dentro se localizan 4-5 cordones de xilema que alternan con los del floema y rodean la médula (Fig. 4A). Las raíces de 0,5-0,7 $\mathrm{mm}$ diámetro, ya poseen estructura secundaria con el centro esclerificado. No se observaron reservorios o conductos secretores en ninguno de los estados de crecimiento.

Rizoma: se analizaron rizomas con un diámetro de 2-4 mm; el corte transversal mostró desde la periferia hacia el centro, la epidermis notablemente pubescente, una o dos capas de hipodermis cuyas células presentan paredes suberificadas (Fig. 4B), rodeando la corteza formada por 18-20 estratos de parénquima, con la capa interna diferenciada en endodermis con 


\begin{tabular}{|c|c|c|c|c|c|c|c|c|c|c|}
\hline C: 11 & C: 1 & C: 2 & C: 3 & C: 4 & C: 5 & C: 6 & C: 7 & C: 8 & C: 9 & C: 10 \\
\hline TE & I & II & III & IV & V & $\mathrm{VI}$ & VII & VIII & IX & $x$ \\
\hline $\mathrm{EA}(\mathrm{mL})$ & 1 & 2 & 3 & 4 & 5 & 6 & 7 & 8 & 9 & 10 \\
\hline $\mathrm{AD}(\mathrm{mL})$ & 9 & 8 & 7 & 6 & 5 & 4 & 3 & 2 & 1 & 0 \\
\hline
\end{tabular}

Referencia: C: Columna.

\begin{tabular}{|ccc|}
\hline \multicolumn{3}{|c|}{ Tabla 2. Valores medios mensuales de } \\
Temperatura $\left({ }^{\circ} \mathrm{C}\right)$ y Lluvias $(\mathrm{mm}) .{ }^{*}$ Meses de \\
recolección de material fresco y determinación de \\
saponinas.
\end{tabular}

banda de Caspary que rodea los haces colaterales. Estos están formados por xilema interno y floema externo con conspicuo casquete de fibras liberianas. Los haces están separados por radios de parénquima que comunican la corteza con la amplia médula parenquimática. Se hallaron 30-34 conductos secretores esquizógenos conteniendo resinas en su interior y gotas de aceite en las células secretoras que forman el epitelio de la cavidad; los cuales se observaron en la corteza dispuestos en dos ciclos: uno interno próximo a la endodermis y otro en la parte media del parénquima cortical (Fig. 4C). En algunos rizomas se observó la diferenciación de esclereidas en el parénquima cortical y medular (Fig. 4D).

Tallo: se estudiaron tallos con diámetro de 2-3 $\mathrm{mm}$ que presentan contorno lobulado y estructura primaria con células epidérmicas cuadrangulares y paredes engrosadas, la pared periclinal externa cubierta por una cutícula delgada; internas a la epidermis se ubican en los lóbulos, 6-8 capas de colénquima angular-lagunar que alternan con clorénquima en los sectores interlobulares; hacia el interior el parénquima cortical está formado por 12-14 capas, la más interna diferenciada en endodermis con banda de Caspary que rodea la eustela con amplia médula parenquimática. Los tallos con un diámetro de 3,0-3,5 $\mathrm{mm}$ se diferencian de los juveniles en el contorno circular, en las células del colénquima con mayor engrosamiento primario de las paredes tangenciales formando un colénquima laminarlagunar y estructura secundaria del tejido vascular (Fig. 4E). Los 18 a 24 conductos secretores, en cualquier estado de crecimiento del tallo, se localizan en el parénquima cortical, algunos junto a la endodermis y predominantemente en la parte media de la corteza; éstos últimos dispuestos en número de uno o de dos a un lado o ambos lados de los haces vasculares (Fig. 4E). Una vista detallada de estos conductos muestra las gotas de aceites esenciales contenidas en las células epiteliales del mismo (Fig. 4F).

Hoja: lámina: ambas epidermis en vista superficial, presentan células poligonales con paredes anticlinales onduladas y numerosos estomas anomocíticos y anisocíticos (Fig. 5A). Se hallaron tricomas glandulares y eglandulares simples, uniseriados, pluricelulares. Los tricomas eglandulares son numerosos y se hallan distribuidos sobre ambas caras de la lámina y en sus bordes; caracterizados por una base de células 

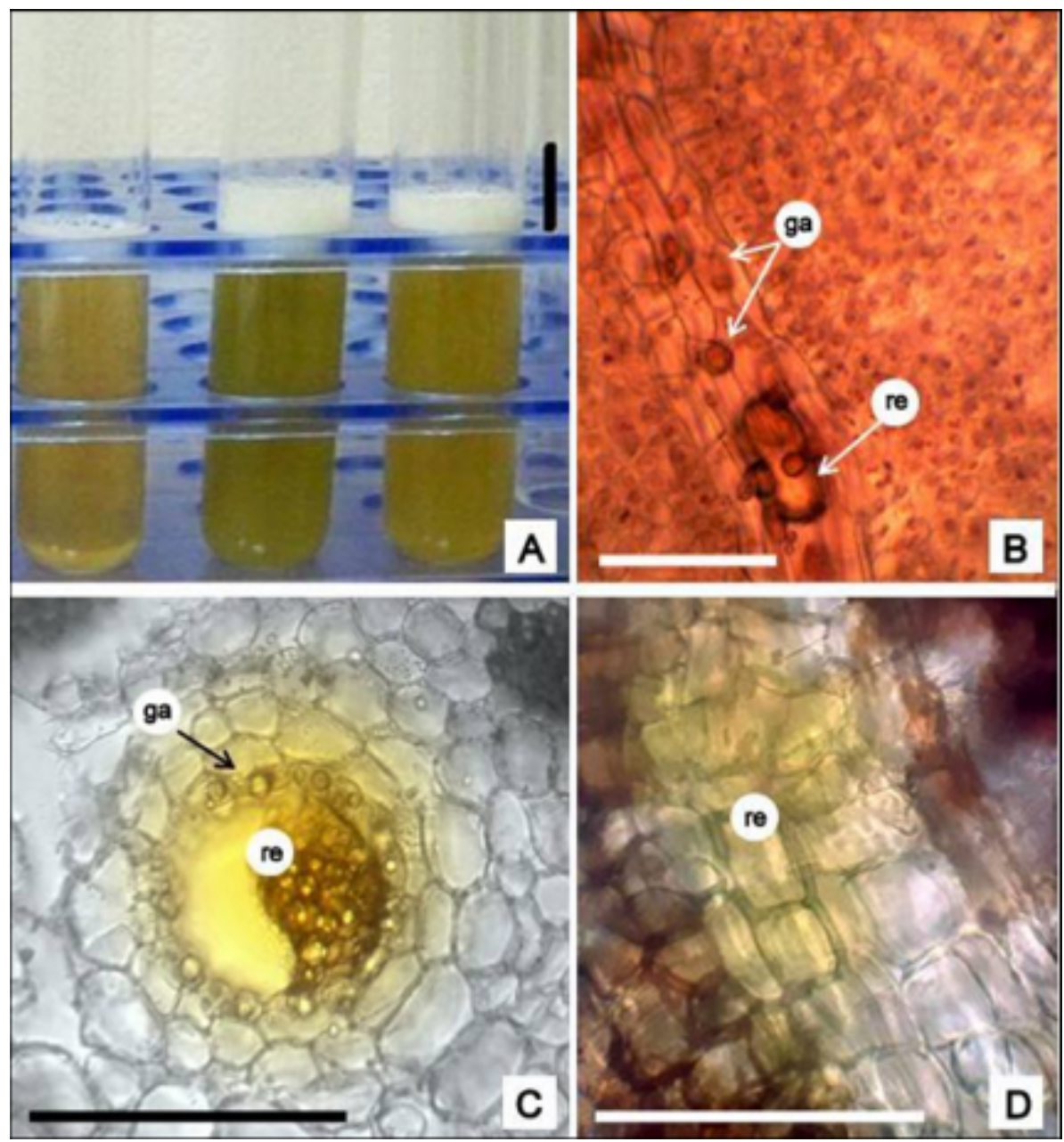

Fig. 3. Saponinas, resinas y aceites en Pascalia glauca. A: Muestra de columnas de saponinas inferiores a $1 \mathrm{~cm}$ de altura. B: Vista paradermal de un conducto secretor en la hoja. C, D: Test positivo para resinas en rizoma C: corte transversal de conducto mostrando resinas en su interior y gotas de aceites en el epitelio secretor; $\mathbf{D}$ : corte longitudinal del mismo conducto. Abreviaturas= ga: gotas de aceites; re: resinas. Escalas= A: $1 \mathrm{~cm}$; B: $100 \mu \mathrm{m}$; C, D: $200 \mu \mathrm{m}$.

epidérmicas radiadas y predominantemente tres células expuestas, la basal ancha, la célula media de longitud variable y la célula apical triangular y aguda, con paredes gruesas y la totalidad de la superficie es groseramente verrucosa (Fig. 5B); en su interior el test resultó positivo para aceites (Fig. 5C). Los tricomas glandulares están formados por 4-10 células de paredes delgadas, la célula terminal oblonga, obtusa; se observaron gotas de aceite en el interior de las células (Fig. 5D).
En el corte transversal la vena media es biconvexa con colénquima angular dispuesto en 4-5 capas hacia la cara adaxial y 2 capas hacia la abaxial; en el centro hay un haz colateral mayor y dos o más haces menores circundados por parénquima. Dos conductos secretores se ubican hacia la cara abaxial sobre ambos lados $\mathrm{y}$ frente al floema del haz vascular central; cuando aparecen conductos secretores frente a los haces menores, se hallan hacia la cara adaxial, enfrentando el xilema (Fig. 5E). Las 


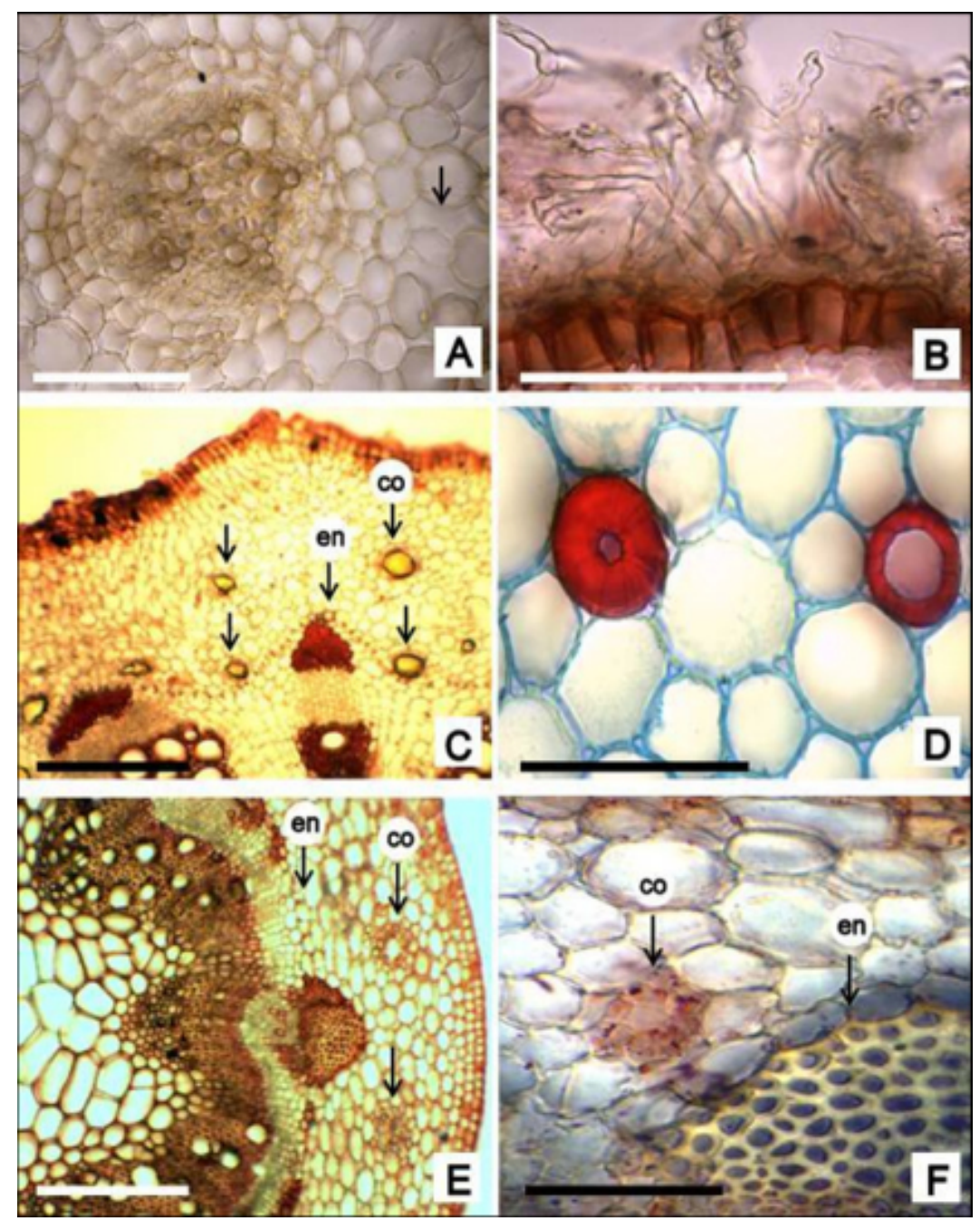

Fig. 4. Estructura de los órganos vegetativos de Pascalia glauca. A: Raíz. Corte transversal mostrando el parénquima cortical con cámaras aeríferas (flecha), endodermis, periciclo y los cordones de xilema y floema rodeando la médula. B-D: Rizoma. B: Detalle de la epidermis formada por células con paredes delgadas y numerosos pelos absorbentes y una capa de hipodermis de células cuadrangulares con paredes suberificadas. C: Sector del rizoma en corte transversal mostrando los dos ciclos de conductos secretores, corticales y próximos a la endodermis. D: Presencia de esclereidas en el parénquima medular. E-F: Tallo. E: Sector del tallo con estructura secundaria mostrando los conductos secretores corticales. F: Test positivo con Oil Red "O", mostrando las gotas de aceite en las células epiteliales de un conducto próximo a la endodermis. Abreviaturas $=$ co: conducto secretor; en: endodermis. Escalas $=$ A, B, D, F: $100 \mu \mathrm{m}, \mathrm{C}, \mathrm{E}: 400 \mu \mathrm{m}$.

epidermis son unistratas, formadas por células cuadrangulares a rectangulares ligeramente mayores en la cara adaxial que en abaxial, con estomas ubicados a nivel o algunos ligeramente hundidos con respecto a las restantes células epidérmicas; en el mesofilo isolateral se localizan los haces vasculares menores rodeados por una notable vaina parenquimática y algunos de mayor tamaño presentan un conducto secretor del lado adaxial, frente al xilema (Fig. 5F, G). Así, en las hojas el número de reservorios varía entre 2-4 hasta 6-7. 


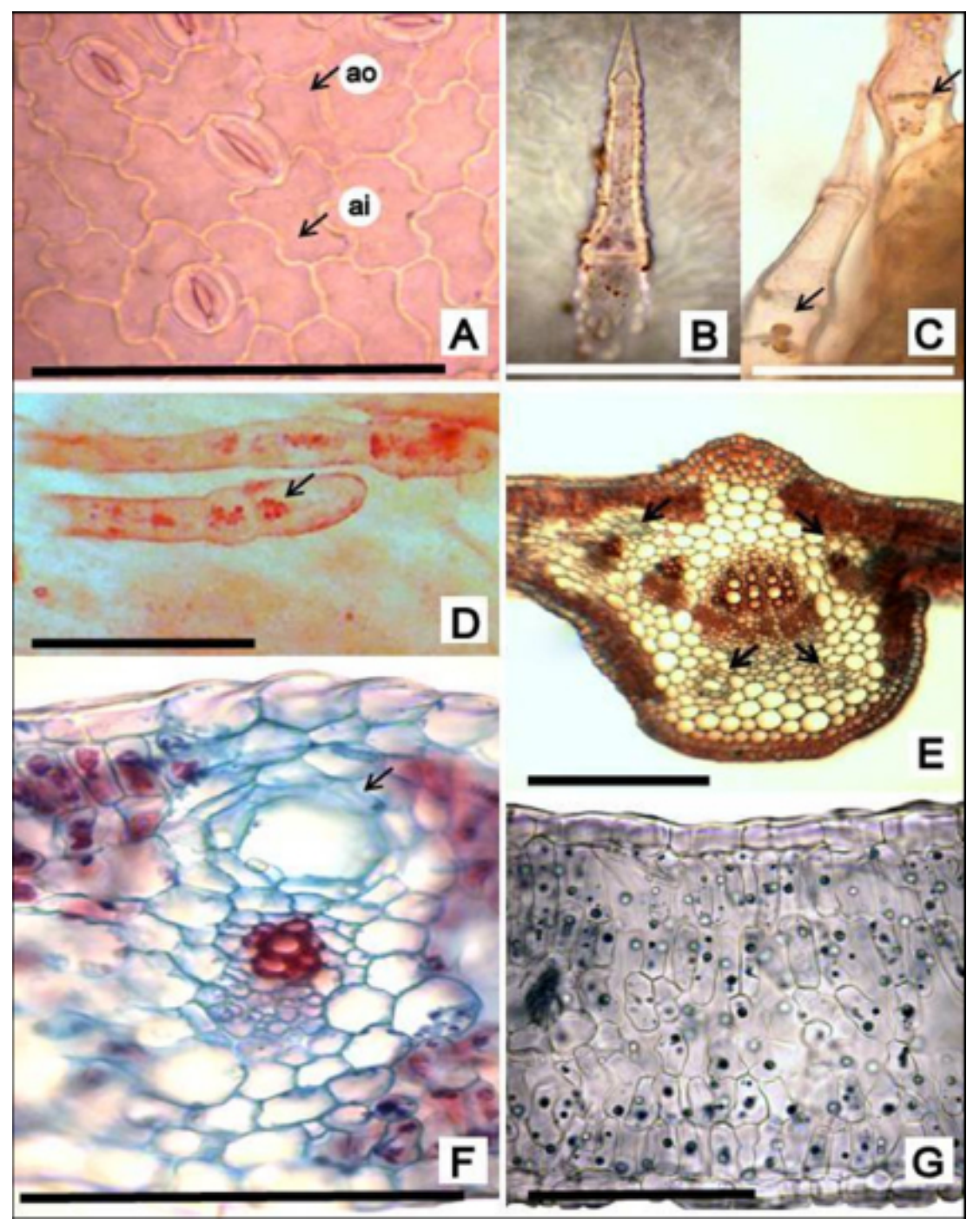

Fig. 5. Estructura de la hoja de Pascalia glauca. A: Epidermis en vista superficial, cara abaxial con estomas anomocíticos y anisocíticos. B, C: Tricomas eglandulares. B: Vista de su pared externa con verrugosidad de ceras; C: Vista de gotas de aceite en su interior. D: Tricomas glandulares con gotas de aceite en sus células. E-G: Cortes transversales. E: Vena media biconvexa, se destaca el haz vascular medio con dos conductos secretores abaxiales y haces colaterales menores con conducto secretor adaxial (flechas). F: Detalle de un conducto secretor adaxial de haces vasculares mayores, en las alas de la lámina. G: Mesofilo isolateral formado por células alargadas dispuestas de manera laxa en la parte media. Abreviaturas= ao: anomocíticos; ai: anisocíticos. Escalas= A-D, G: $50 \mu \mathrm{m}$; E: $400 \mu \mathrm{m} ; \mathrm{F}: 25 \mu \mathrm{m}$.

\section{Órganos reproductivos}

Filarios y pálea: estas partes de la inflorescencia clarificadas permitieron observar por transparencia varios conductos secretores longitudinales, así como en el borde numerosos tricomas con abundantes gotas de aceite (Fig. 6A, a, B).
Flor ligulada femenina (Fig. 6C): el test para lípidos dio reacción positiva en todas las partes de la flor, resultando notable en la corola las células conteniendo gotas de aceites (Fig. 6D).

Flor tubulosa hermafrodita (Fig. 6E): el test para lípidos dio reacción positiva en todas las partes de 


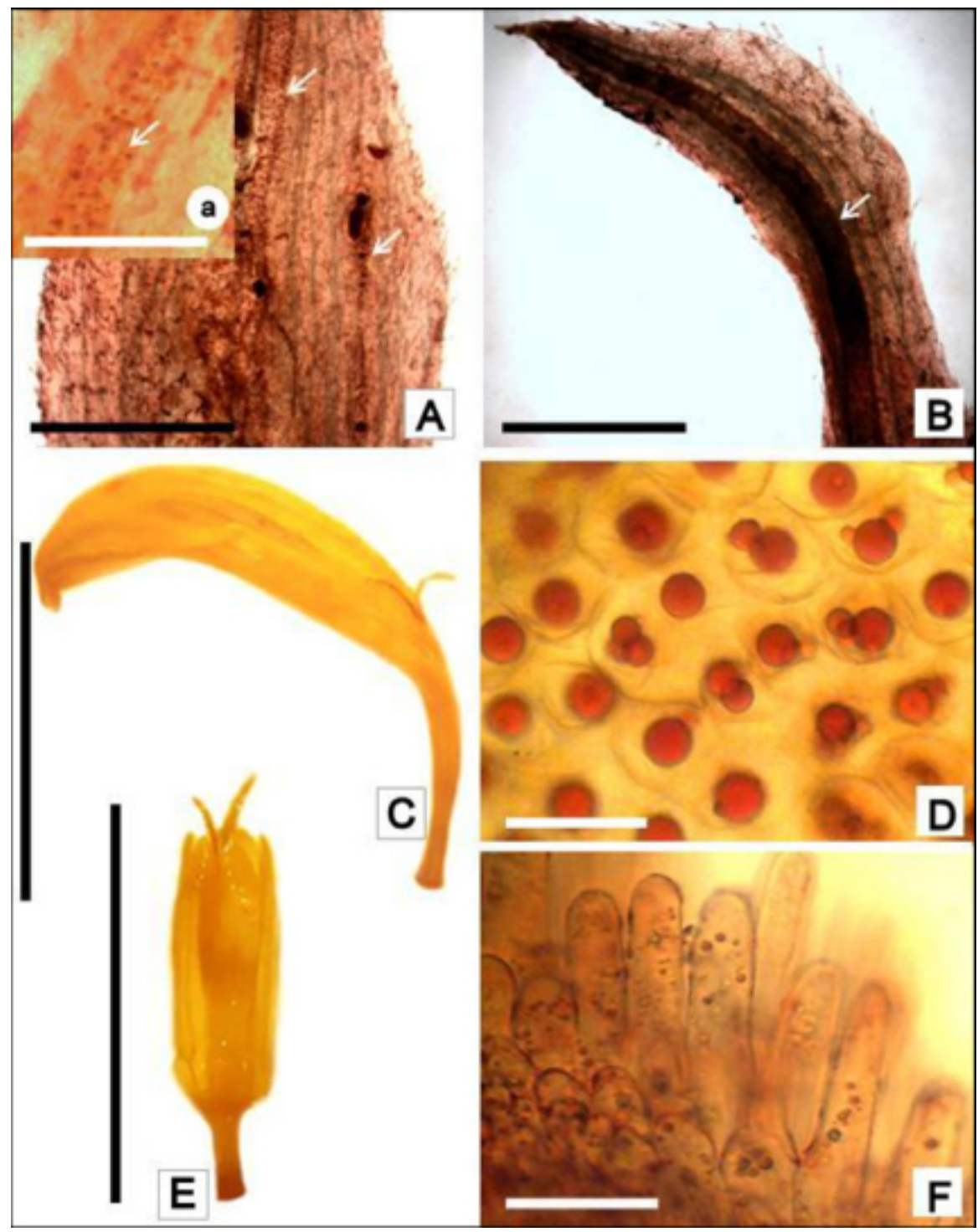

Fig. 6. Partes de la inflorescencia de Pascalia glauca. A, a, B: Filario y Pálea. A: parte del filario mostrando conductos secretores longitudinales y pubescencia; $\mathbf{a}$ : detalle de las gotas de aceite en un conducto (flecha). B: Pálea con un conducto secretor dorsal importante (flecha); en el borde, pelos eglandulares con aceites esenciales. C-F: Flores. C: Flor ligulada. D: Detalle de las gotas de aceite en células de la corola. E: Flor tubulosa. F: Detalle de las papilas estigmáticas con gotas de aceites esenciales. Escalas= A, B: $1 \mathrm{~mm}$; a: $400 \mu \mathrm{m}$; C, E: $5 \mathrm{~mm}$; D, F: $50 \mu \mathrm{m}$.

la flor, presentando las células de la corola con gotas de aceite como en la flor ligulada; en los estambres las gotas de aceite se hallaron principalmente en el filamento y conectivo y en el gineceo, en el ovario, estilo y estigma, incluidas las papilas estigmáticas (Fig. 6F).

\section{Discusión y Conclusiones}

La cantidad de saponinas halladas en los diferentes órganos fue ligeramente positiva coincidiendo con Rondina et al. (1970), quienes solo realizaron la determinación en las hojas y también hallaron 
escasa cantidad de este compuesto. En el presente estudio, si bien su contenido resultó escaso, se determinó que varía con la estación del año. Así, en las hojas la mayor cantidad está presente en el período de primavera alcanzando la columna de espuma la máxima altura $(7 \mathrm{~mm})$, justamente cuando la planta está en pleno crecimiento. Este resultado coincide con el período de diciembre señalado por Correa et al. (2017) como uno de los de mayor toxicidad para el ganado. En los tallos, la mayor altura de la columna de espuma ( $4 \mathrm{~mm}$ ), correspondió al verano-otoño, período del año en que las plantas se encuentran en floración y la presencia de saponinas se vería potenciada en su poder de toxicidad por otros compuestos como resinas y principalmente los aceites esenciales de los capítulos. Este resultado coincide con el período de marzo señalado por Correa et al. (2017) como de máxima toxicidad.

Los conductos secretores de resinas se encontraron en todos los órganos vegetativos (excepto en la raíz) mientras que hasta ahora se habían mencionado solo en hojas y rizomas (Burkart \& Carera, 1953; Reid Lorna \& Lagomarsino, 2009). De acuerdo con la bibliografía, los diterpenos son componentes de pigmentos o de resinas, siendo que la toxicidad es atribuida a un diterpeno (atractilósido) (Schteingart \& Pomilio, 1984; Casabuono \& Pomilio, 2000; Micheloud \& Odriozola, 2012), las resinas quedarían implicadas en el proceso de toxicidad. Los aceites esenciales son compuestos aromáticos e insolubles en agua y están compuestos por muchas sustancias químicas complejas, entre ellas los terpenos, los que son uno de los constituyentes principales de los aceites esenciales de algunas plantas y flores. Dada la característica tóxica de estos aceites, es posible también interactúen con el atractilósido y las resinas. Los aceites esenciales se hallaron en gran cantidad en las inflorescencias, los mismos ya han sido ensayados como repelentes (Rufinnengo et al., 2005) y se obtuvieron resultados preliminares altamente positivos en un ensayo de control de nemátodos (Rípodas et al., 2018). De acuerdo con los resultados, la acción tóxica estaría dada por la acción conjunta de diversos compuestos hallados en los órganos de la planta. Por otro lado, la abundancia de aceites esenciales en las inflorescencias la señalan como una especie promisoria para su utilización en el control de plagas agrícolas.

\section{Contribución DE los AUtores}

Los autores han contribuido por igual en la producción del trabajo, desde el diseño, la realización de las tareas, obtención de datos e interpretación, como la preparación del manuscrito.

\section{Agradecimientos}

Al personal del Departamento de Sismología e Información Meteorológica de la Facultad de Ciencias Astronómicas y Geofísicas, UNLP por proveernos los registros climáticos y a los evaluadores por sus valiosas sugerencias.

\section{Bibliografía}

ARAMBARRI, A. M. 2018. La "técnica de clarificación 5-5-5", un método natural para el tratamiento de material vegetal. Bol. Soc. Argent. Bot. 53: 579-586. https://dx.doi.org/10.31055/1851.2372.v53.n4.21980

BAILAC, P. N., A. D. DELLACASA, M. I. PONZI \& N. H FIRPO. 2005. Essential oil composition of Wedelia glauca (Ort.) Hoffman ex Hicken from Argentina. J. Essent. Oil Res. 17: 401-402. https://doi.10.1080/10412905.2005.9698942

BOELCKE, O. 1981. Plantas vasculares de la Argentina, nativas y exóticas. Fundación para la Educación, la Ciencia y la Cultura, Buenos Aires.

BURKART, A. \& M. N. CARERA. 1953. Estudios sobre malezas. Morfología vegetativa y germinación del sunchillo (Wedelia glauca). Darwiniana 10: 113-144.

CABRERA, A. L. 1963. Wedelia glauca. Compuestas. En: CABRERA, A. L. (ed.), Flora de la provincia de Buenos Aires. Colecc. Ci. Inst. Nac. Tecnol. Agropecu. 4: 206-207.

CABRERA, A. L. \& A. BURKART. 1974. Wedelia glauca. Compuestas. En: BURKART A. (dir.), Flora ilustrada de Entre Ríos. Colecc. Ci. Inst. Nac. Tecnol. Agropecu. 6: 353-355.

CASABUONO, A. C. \& A. B. POMILIO. 2000. Toxicidad en vegetales: problemática y análisis. SAIPA - Sociedad Argentina para la Investigación de Productos Aromáticos. Noveno Congreso Nacional de recursos naturales, aromáticos y medicinales. Anales de SAIPA 6: 135-146.

CORREA, R. J., J. I. MASCHIO \& M. L. MASCAREÑO VARAS. 2017. Las plantas tóxicas en la ganadería, 


\section{P. Hernández y A. M. Arambarri -Anatomía y metabolitos secundarios de Pascalia glauca}

Wedelia glauca (Ort.) Hoffmann ex Hicken. Facultad de Ciencias Agrarias, Universidad Nacional de Catamarca. Revista de Divulgación Técnica 73: $1-15$.

COSA, M. T., N. DOTTORI, L. STIEFKENS, M. HADID, M. MATESEVACH, N. DELBÓN, P. WIEMER, S. MACHADO, V. CABRERA, C. COSTA, A. PÉREZ \& A. TRENCHI. 2014. Aplicación de técnicas de histología vegetal a la resolución de diversos problemas. Laboratorio de Morfología Vegetal, Universidad Nacional de Córdoba.

DE LA PEÑA, M. R. \& J. F. PENSIERO. 2004. Plantas argentinas. Catálogo de nombres comunes. Ed. L.O.L.A., Buenos Aires.

DEL VitTo, L. A. \& E. M. PETEnATti. 2015. Asteráceas de importancia económica y ambiental. Segunda parte: otras plantas útiles y nocivas. Multequina 24: 47-74.

GALLO, G. 1979. Plantas tóxicas para el ganado en el cono sur de América. Ed. Universitaria de Buenos Aires.

GALLO, G. 1987. Plantas tóxicas para el ganado en el cono sur de América. 2da ed. Hemisferio Sur, Buenos Aires.

GIMÉNEZ, G. D. 2017. Plantas tóxicas para rumiantes en pastoreo. Sitio Argentino de Producción Animal. Disponible en: http://www.produccion-animal.com. ar [Acceso: 06 Enero 2019].

GURR, E. 1971. Synthetic dyes in biology, medicine and chemistry $1^{\text {st }}$. edition. Academic Press, London.

HARBORNE, J. B. 1989. Phytochemical methods: a guide to modern techniques of plant analysis. 3ra. ed., Chapman \& Hall, NY.

https://doi: 10.1007/978-94-009-5921-7

HIERONYMUS, J. 1882. Plantae diaphoricae florae argentinae. Bol. Acad. Nac. Cien., Córdoba 4: 199598.

JOHANSEN, D. A. 1940. Plant microtechnique. Mc Graw Hill Book Company, Inc. New York.

MARZOCCA, A. 1957. Manual de malezas. 1ra. Edición. Imprenta y casa editora CONI, Buenos Aires. 530 pp.

MARZOCCA, A., O. J. MÁRSICO \& O. DEL PUERTO. 1986. Manual de malezas. 3ra. Reimpresión. Ed. Hemisferio Sur S.A., Buenos Aires.

MICHELOUD, J. F. \& E. ODRIOZOLA. 2012. Actualización sobre la intoxicación por Wedelia glauca (Ort.) Hoffm. Ex Hicken, Asteraceae. Revista FAVE-Ciencias Veterinarias 11: 31-42.
MONTALDI, E., J. BELTRANO, W. ABEDINI \& T. MARRONE. 1984. Factores que afectan la formación de rizomas en el "sunchillo" [Wedelia glauca (Ort.) Offm]. Revista Fac. Agron. Univ. Nac. La Plata 5: 139-145.

PLATANOW, N. S. \& T. A. LÓPEZ. 1978. Wedelia glauca, estudios sobre su toxicidad. Balcarce, Buenos Aires. Revista Argentina de Producción Animal 6: 620-625.

RAGONESE, A. E. \& V. A. MILANO. 1984. Vegetales y sustancias tóxicas de la flora argentina. En: Enciclopedia Argentina de agricultura y jardinería. 2da. Ed. Vol. 2: 354-357. Ed. Acmé S.A.C.I., Buenos Aires.

REID LORNA, C. \& E. D. LAGOMARSINO. 2009. Intoxicación por Cestrum parqui y Wedelia glauca en ganado bovino: epidemiología, prevención $y$ control, pp. 478-485. Avances en la producción vegetal y animal del NOA (2007-2009), Tucumán.

RÍPODAS, J. I., S. GARITA, V. BERNARDO, M. RUSCITTI, M.C.ARANGO \& M. P. HERNÁNDEZ. 2018. Efecto de la aplicación in vitro de aceites esenciales sobre Nacobbus aberrans y Paecilomyces lilacinus. Dominguezia 34: 112-113.

RONDINA, R. V. D., M. E. MENDIONDO \& J. D. COUSSIO. 1970. Estudio fitoquímico de plantas medicinales argentinas. II. RIA, INTA, Serie 2, Biología y producción vegetal 7: 271-276.

RUFFINENGO, S., M. EGUARAS, I. FLORIS, C. FAVERIN, P. BAILAC \& M. PONZI. 2005. $\mathrm{LD}_{50}$ and repellent effects of essential oils from Argentinian wild plant species on Varroa destructor. J. Econ. Entomol. 98: 651-655. https://doi.org/10.1603/0022-0493-98.3.651.

SCHTEINGART, C. D. \& A. B. POMILIO. 1984. Atractyloside, toxic compound from Wedelia glauca. J. Nat. Prod. 47: 1046-1047. doi: 10.1021/np50036a029

TROIANI, H. O. 2016. Wedelia glauca. En: FERNÁNDEZ, O. A., E. S. LEGUIZAMÓN \& H. A. ACCIARESI (eds.), Malezas e invasoras de la Argentina. Descripción y reconocimiento. T 2, pp. 314. Editorial Universidad Nacional del Sur (Ediuns), Bahía Blanca, Buenos Aires.

ZULOAGA, F. O., O. MORRONE \& M. J. BELGRANO (eds.). 2008. Catálogo de las plantas vasculares del Cono Sur (Argentina, Sur de Brasil, Chile, Paraguay y Uruguay). II. Monogr. Syst. Bot. Missouri Bot. Gard. 107: 1905-1908. Disponible en: http://www. darwin.edu.ar/ [Acceso: 5 diciembre 2018]. 
